KCL-MTH-02-08

hep-th//0204207

\title{
Massive IIA Supergravity as a Non-linear Realisation
}

\author{
Igor Schnakenburg and Peter West \\ Department of Mathematics \\ King's College, London, UK
}

\begin{abstract}
A description of the bosonic sector of massive IIA supergravity as a non-linear realisation is given. An essential feature of this construction is that the momentum generators have non-trivial commutation relations with the generators associated with the gauge fields.
\end{abstract}

Igor Schnakenburg is financially supported by DAAD (D/00/09914).

email: schnake, pwest@mth.kcl.ac.uk 


\section{Introduction}

Long ago Nahm [1] pointed out that supergravity theories could only exist in eleven and less space-time dimensions and that the maximum number of supersymmetries they could possess were contained in spinors that had in total no more than 32 real components. The supergravity theories with 32 components are called maximal supergravity theories. There is a unique such theory in eleven dimensions which was constructed [2] as an application of the Noether method that was used to construct the first supergravity theory, the $N=1, D=4$ supergravity theory. However, in ten dimensions there are two such maximal supergravity theories called IIA and IIB. These two theories possess different supersymmetries which when decomposed in terms of Majorana-Weyl spinors are of opposite and the same chirality respectively. While the construction of the IIA theory [3] was found by dimensional reduction of the eleven dimensional supergravity theory, the construction of the IIB theory $[4,5,6]$ required new techniques. There is a modification of the IIA theory that preserves the number of supersymmetries, but introduces a dimensionfull parameter [7]. This theory is called massive IIA supergravity and it possesses a cosmological constant.

One of the most remarkable features of supergravity theories is that the scalars in the supergravity multiplets always occur in a coset structure [8]. While this can be viewed as a consequence of supersymmetry, the groups that occur in these cosets are rather mysterious $[9,10,11]$. It has been conjectured [12] that the symmetries found in these cosets are symmetries of the associated non-perturbative string theory.

The coset construction for the description of the scalars was extended to include the gauge fields for the maximal supergravity theories [13]. In this construction all gauge fields and scalars were introduced along with their duals. The advantage of this approach is that the equations of motion can be reduced to first order equations in form of a generalised selfduality condition. This method was subsequently applied to massive IIA [14]. However, in these papers the vector indices on the gauge fields did not arise from the underlying group, but were introduced by hand. As such, it is difficult to see how this construction could be extended to include the other degrees of freedom of the theory, namely the graviton and the fermions.

Recently, it was shown that the entire bosonic sectors, including gravity, of the eleven dimensional supergravity theory and the ten dimensional IIA [15] and IIB supergravity theories [16], could be formulated as a non-linear realisation. It was also conjectured, that when suitably formulated eleven dimensional supergravity would be invariant under a Kac-Moody algebra [17]. Although, this conjecture was not proved in [17] some evidence was given and the Kac-Moody algebra was identified. It had rank eleven and was denoted $E_{11}$ [17]. A similar analysis found that $E_{11}$ was also the Kac-Moody algebra that would underlie the ten dimensional IIA and IIB theories $[16,17]$. As has been pointed out by one of the authors $(\mathrm{PCW})$ in a number of seminars it could be that $E_{11}$ is part of an even 
larger underlying algebra that is a Borcherds algebra.

This is consistent with the general belief that all these theories are part of a larger theory which has been called $\mathrm{M}$ theory [18]. It was conjectured [17] that $E_{11}$ over an appropriate field was a symmetry of $M$ theory. However, as seen from this perspective M theory does not necessarily live in eleven dimensions, but rather has its dimension undetermined. A particular theory results from a choice of "vacuum" in M theory and the dimension of the resulting theory is a consequence of the Lorentz group that is contained in the subgroup of $E_{11}$ that is preserved by the "vacuum" under consideration.

In this paper we extend some of these considerations to the massive IIA theory and as a first step show that its entire bosonic sector can be described as a non-linear realisation.

\section{Massive IIA Supergravity}

The bosonic sector of massive IIA supergravity was originally [7] described in terms of the same fields as in the ten-dimensional chiral theory without the cosmological constant. These fields were the graviton, $h_{a}{ }^{b}$, the scalar $A$ (dilaton) and the $p$-forms $A_{a_{1} \cdots a_{p}}$ for $p=1,2,3$. However, the massive theory also contains a constant which is related to the cosmological constant. The bosonic part of the original Lagrangian given by Romans in [7] was

$$
\begin{aligned}
e^{-1} L=R & -\frac{1}{2} \partial_{\mu} A \partial^{\mu} A-\frac{1}{12} e^{-1 / 2 A} F^{\mu \nu \rho \sigma} F_{\mu \nu \rho \sigma}-\frac{1}{3} e^{A} G^{\mu \nu \rho} G_{\mu \nu \rho}-m^{2} e^{-3 / 2 A} B^{\mu \nu} B_{\mu \nu} \\
& -\frac{1}{2} m^{2} e^{-5 / 2 A}+\frac{e^{-1}}{6 \cdot 48}\left(\epsilon ^ { \mu \nu \rho \sigma \mu _ { 1 } \cdots \mu _ { 6 } } \left(16 \partial_{\mu} A_{\nu \rho \sigma}^{\prime} \partial_{\mu_{1}} A_{\mu_{2} \mu_{3} \mu_{4}}^{\prime} B_{\mu_{5} \mu_{6}}\right.\right. \\
+ & \left.16 m \partial_{\mu} A_{\nu \rho \sigma} B_{\mu_{1} \mu_{2}} B_{\mu_{3} \mu_{4}} B_{\mu 5 \mu_{6}}+\frac{36}{5} m^{2} B_{\mu \nu} B_{\rho \sigma} B_{\mu_{1} \mu_{2}} B_{\mu_{3} \mu_{4}} B_{\mu_{5} \mu_{6}}\right)
\end{aligned}
$$

where

$$
G_{\mu \nu \rho}=3 \partial_{[\mu} B_{\nu \rho]}, \quad \text { and } \quad F_{\mu \nu \rho \sigma}=4\left(\partial_{[\mu} A_{\nu \rho \sigma]}^{\prime}+6 m B_{[\mu \nu} B_{\rho \sigma]}\right) .
$$

By redefining the fields according to

$$
B_{\mu \nu}=A_{\mu \nu}+\frac{2}{m} \partial_{[\mu} A_{\nu]} \quad A_{\mu \nu \rho}^{\prime}=A_{\mu \nu \rho}-6 A_{[\mu} A_{\nu \rho]}-\frac{6}{m} A_{[\mu} \partial_{\mu} A_{\rho]},
$$

as explained in [19], it can be rewritten in the form

$$
\begin{gathered}
e^{-1} L=R-\frac{1}{2} \partial_{\mu} A \partial^{\mu} A-\frac{1}{12} e^{1 / 2 A} F_{\mu \nu \rho \sigma} F^{\mu \nu \rho \sigma}-\frac{1}{3} e^{-A} G_{\mu \nu \rho} G^{\mu \nu \rho}-e^{3 / 2 A} F_{\mu \nu} F^{\mu \nu} \\
-\frac{1}{2} m^{2} e^{5 / 2 A}+\frac{e^{-1}}{18} \epsilon^{\mu_{1} \cdots \mu_{10}}\left(\partial_{\mu_{1}} A_{\mu_{2} \mu_{3} \mu_{4}} \partial_{\mu_{5}} A_{\mu_{6} \mu_{7} \mu_{8}} A_{\mu_{9} \mu_{10}}+m \partial_{\mu_{1}} A_{\mu_{2} \mu_{3} \mu_{4}} A_{\mu_{5} \mu_{6}} A_{\mu_{7} \mu_{8}} A_{\mu_{9} \mu_{10}}\right.
\end{gathered}
$$




$$
\left.+\frac{9}{20} m^{2} A_{\mu_{1} \mu_{2}} A_{\mu_{3} \mu_{4}} A_{\mu_{5} \mu_{6}} A_{\mu_{7} \mu_{8}} A_{\mu_{9} \mu_{10}}+18 m \partial_{\mu_{1}} A_{\mu_{2} \cdots \mu_{10}}\right),
$$

where the gauge invariant field strengths are now given by

$$
\begin{gathered}
F_{\mu \nu}=2\left(\partial_{[\mu} A_{\nu]}+\frac{1}{2} m A_{\mu \nu}\right), \text { and } G_{\mu \nu \rho}=3 \partial_{[\mu} A_{\nu \rho]}, \\
F_{\mu \nu \rho \sigma}=4\left(\partial_{[\mu} A_{\nu \rho \sigma]}+6 A_{[\mu} \partial_{\nu} A_{\rho \sigma]}+\frac{3}{2} m A_{[\mu \nu} A_{\rho \sigma]}\right) .
\end{gathered}
$$

The terms containing negative powers of $m$ form a total divergence and can be dropped. In this second formulation the one form gauge field, which had been absorbed in the two form gauge field to make it massive in the former formulation, appears explicity and one can take $m \rightarrow 0$ to find the Lagrangian of massless IIA supergravity in a straightforward way.

Following [19], in this formulation we may treat $m$ as a dynamical field. The field equation for the new nine form gauge fields states that $\partial_{\mu} m=0$ i.e. $m$ is a constant. While the field equation for $m$ sets the field strength of the nine form gauge plus a combination of other forms in the theory equal to the epsilon symbol. We note that unlike for the other gauge fields these equations are first order. In [19] it was argued that one can derive the field equations for the purely bosonic sector of massive IIA supergravity from a Lagrangian which does not contain the mass parameter $m$ at all. To obtain this formulation the field equation for $m$ was plugged back into the Lagrangian (1.4). The advantage of this approach, as was explained in [19] is that this theory then naturally couples to an eight brane.

References [13] and [14] introduced duals of all the original gauge fields. As a result, the field equations for the gauge potentials could be reduced to first order. In the case of massive IIA supergravity [14] the dual of the nine form gauge field was taken to be a "minus one form" whose field strength was then dual to the ten form field strength of the nine form gauge field. We have noted in the last paragraph that the field equation for the nine form gauge potential is necessarily first order. We therefore do not introduce a dual of the nine form potential. In this paper we will introduce dual gauge fields for all the original gauge field, namely $A_{a_{1} \cdots a_{q}}$ for $q=5,6,7,8$, but not for the nine form gauge field. We will find that the momentum operator plays an important role in place of the generator associated with the minus one form. Although to see the full symmetry one will have to introduce "duals of gravity" we will not do this here. The complete bosonic field content we require is thus given by:

$$
h_{a}^{b}, A, A_{c}, A_{c_{1} c_{2}}, A_{c_{1} c_{2} c_{3}}, A_{c_{1} \ldots c_{5}}, A_{c_{1} \ldots c_{6}}, A_{c_{1} \ldots c_{7}}, A_{c_{1} \ldots c_{8}}, A_{c_{1} \ldots c_{9}} .
$$

In a non-linear realisation these fields are considered as the Goldstone bosons. We therefore introduce the corresponding generators

$$
K_{b}^{a}, R, R^{c}, R^{c_{1} c_{2}}, R^{c_{1} c_{2} c_{3}}, R^{c_{1} \ldots c_{5}}, R^{c_{1} \ldots c_{6}}, R^{c_{1} \ldots c_{7}}, R^{c_{1} \ldots c_{8}}, R^{c_{1} \ldots c_{9}} .
$$


The generators $K^{a}{ }_{b}$ satisfy the commutation relations of $G L(10, \mathbf{R})$ and the non-zero commutation relations between all generators mentioned above are given by

$$
\begin{gathered}
{\left[K_{b}^{a}, K_{d}^{c}\right]=\delta_{b}^{c} K_{d}^{a}-\delta_{d}^{a} K_{b}^{c}, \quad\left[K_{b}^{a}, P_{c}\right]=-\delta_{c}^{a} P_{b}, \quad\left[K_{b}^{a}, R^{c_{1} \cdots c_{p}}\right]=\delta_{b}^{c_{1}} R^{a c_{2} \cdots c_{p}}+\cdots,} \\
{\left[R, R^{c_{1} \cdots c_{p}}\right]=c_{p} R^{c_{1} \cdots c_{p}}, \quad\left[R^{c_{1} \cdots c_{p}}, R^{c_{1} \cdots c_{q}}\right]=c_{p, q} R^{c_{1} \cdots c_{p+q}},}
\end{gathered}
$$

where $+\cdots$ means the appropriate anti-symmetrisations. We also include the momentum generator $P_{a}$ in the symmetry algebra and its non-trivial relations with the other generators are given by

$$
\left[P_{a}, R^{c_{1} \cdots c_{p}}\right]=-m b_{p}\left(\delta_{a}^{c_{1}} R^{c_{2} \cdots c_{p}}+\cdots\right), \quad\left[P_{a}, R\right]=-m b_{0} P_{a} .
$$

In what follows it will often be useful to denote $c_{-1}=m b_{0}$, since in this way one can view the last commutator of equation (1.10) as an extension of those of equation (1.9).

If the coefficients are taken to be

$$
\begin{gathered}
c_{3}=-c_{5}=-\frac{1}{4}, \quad c_{2}=-c_{6}=\frac{1}{2}, \quad c_{1}=-c_{7}=-\frac{3}{4}, \quad c_{9}=-c_{-1}=\frac{5}{4}, \\
c_{1,2}=-c_{2,3}=-c_{3,3}=c_{1,5}=c_{2,5}=2, \quad c_{3,5}=1, \quad c_{2,6}=2, \quad c_{1,7}=3, \\
c_{2,7}=-4, \quad b_{2}=-\frac{1}{2}, \quad b_{7}=-\frac{1}{2}, \quad b_{9}=\frac{5}{8},
\end{gathered}
$$

(all not mentioned coefficients are equal to zero) then we can verify that all Jacobi identities are satisfied. For example, the generators corresponding to the gauge fields (all $c$ 's) fulfill the condition

$$
c_{q, r} c_{p, q+r}=c_{p, q} c_{p+q, r}+c_{p, r} c_{q, p+r}
$$

where $q, p$ and $r$ indicate the rank of the generators. The Jacobi identities which involve $b_{0}$ obey the above relation provided we take $m b_{0}=c_{-1}$ where appropriate. The Jacobi identities that involve the $b_{p}, p \neq 0$ structure constants in the new commutators of equation (1.10) obey the relation

$$
b_{p+q} c_{p, q}=b_{p} c_{p-1, q}+b_{q} c_{p, q-1} .
$$

One such example is given by the Jacobi identity involving $R^{a b}, R^{c_{1} \cdots c_{7}}$ and $P_{d}$ which is satisfied provided $c_{2,7} b_{9}=c_{2,6} b_{7}+b_{2} c_{1,7}$.

The relations of equations (1.9) are the same as the algebra denoted $G_{I I A}$ [16] relevant to the IIA supergravity theory except that they also include the rank nine generator. For the IIA theory the commutators of equation (1.10) vanish. Since the above mentioned commutation relations include those of IIA supergravity [16], we call the modified group which is generated by the above generators $G_{m I I A}$.

We can write a general group element of the corresponding group as

$$
g=\exp \left(x^{\mu} P_{\mu}\right) \exp \left(h_{a}{ }^{b} K^{a}{ }_{b}\right) g_{A} \equiv g_{x} g_{h} g_{A},
$$


where

$$
\begin{gathered}
g_{A}=e^{(1 / 9 !) A_{a_{1} \cdots a_{9}} R^{a_{1} \cdots a_{9}}} e^{(1 / 8 !) A_{a_{1} \cdots a_{8}} R^{a_{1} \cdots a_{8}}} e^{(1 / 7 !) A_{a_{1} \cdots a_{7}} R^{a_{1} \cdots a_{7}}} \times \\
e^{(1 / 6 !) A_{a_{1} \cdots a_{6}} R^{a_{1} \cdots a_{6}}} e^{(1 / 5 !) A_{a_{1} \cdots a_{5}} R^{a_{1} \cdots a_{5}}} e^{(1 / 3 !) A_{a_{1} a_{2} a_{3}} R^{a_{1} a_{2} a_{3}}} e^{(1 / 2 !) A_{a_{1} a_{2}} R^{a_{1} a_{2}}} e^{A_{a_{1}} R^{a_{1}}} e^{A R} .
\end{gathered}
$$

Of course, we could have chosen any other representation, but the calculations turn out to be simplest in this particular exponential representation.

We now construct a non-linear realisation of the $G_{m I I A}$ algebra taking the local subalgebra to be the Lorentz group. As such, we demand that the theory is invariant under

$$
g \rightarrow g_{0} g h^{-1}
$$

where $g_{0}$ is a rigid element from the whole group $G_{m I I A}$ and $h$ is a local Lorentz transformation. We calculate the Maurer-Cartan form

$$
\mathcal{V}=g^{-1} d g-\omega
$$

in the presence of the Lorentz connection $\omega=\frac{1}{2} d x^{\mu} \omega_{\mu b}{ }^{a} J^{b}{ }_{a}$, which transforms as

$$
\omega \rightarrow h \omega h^{-1}+h d h^{-1}
$$

As a result, $\mathcal{V}$ transforms as

$$
\mathcal{V} \rightarrow h \mathcal{V} h^{-1}
$$

We split the Cartan form in gravity and gauge field parts according to

$$
\mathcal{V}=\left(g_{h}^{-1} d g_{h}-\omega\right)+\left(g_{A}^{-1} d g_{A}+g_{A}^{-1}\left(g_{h}^{-1} d g_{h}\right) g_{A}-g_{h}^{-1} d g_{h}\right)
$$

and set the result to be equal to

$$
\mathcal{V} \equiv d x^{\mu}\left(\hat{e}_{\mu}{ }^{a} P_{a}+\Omega_{\mu a}{ }^{b} K^{a}{ }_{b}\right)+d x^{\mu}\left(\sum_{p=1, p \neq 4}^{9} \frac{1}{p !} e^{-c_{p-1} A} \tilde{D}_{\mu} A_{a_{1} \cdots a_{p}} R^{a_{1} \cdots a_{p}}\right)
$$

where

$$
\Omega_{a b}{ }^{c} \equiv\left(\hat{e}^{-1}\right)_{a}^{\mu}\left(\left(e^{-1} \partial_{\mu} e\right)_{b}{ }^{c}-\omega_{\mu b}{ }^{c}\right)
$$

and

$$
\hat{e}_{\mu}^{a}=e^{-5 / 4 A}\left(e^{h}\right)_{\mu}^{a}, \quad e_{\mu}^{a}=\left(e^{h}\right)_{\mu}{ }^{a} .
$$

We see that the object in front of the momentum generator gets altered with respect to IIA (where it was $e_{\mu}{ }^{a}$ ) due to the non-vanishing commutator in equation (1.10). We also see that the vielbeins in the $\Omega_{\mu b}{ }^{c}$ equation (1.21) are the unhatted vielbeins. The additional factor of $e^{-5 / 4 A}$ just multiplies the usual expression of $\Omega_{a b}{ }^{c}$ from the massless case [16]. As we will see in chapter 2 , the physical vielbein therefore remains unchanged. The objects $\tilde{D}_{\mu} A_{a_{1} \cdots a_{p}}$ defined in (1.20) will be explicitly stated below. 
Massive IIA supergravity is the non-linear realisation of the group that is the closure of the $G_{m I I A}$ algebra given above and the conformal group in ten dimensions. We therefore take only those combinations of the Cartan forms of $G_{m I I A}$ that can be rewritten as Cartan forms of the conformal group (see section 2). Lorentz covariant objects which are also covariant under the full non-linear realisation of the closure of the conformal and the $G_{m I I A}$ algebra are then for example the completely anti-symmetrised derivatives

$$
\tilde{F}_{a_{1} \cdots a_{p}}=p e^{-5 / 4 A} e^{-c_{p-1} A} \tilde{D}_{\left[a_{1}\right.} A_{\left.a_{2} \cdots a_{p}\right]},
$$

where we have to use $\tilde{D}_{a}=\hat{e}_{a}{ }^{\mu} \tilde{D}_{\mu}$ to convert the curved index to a flat index groupcovariantly. As the physical vielbein is the unhatted one, we gain an additional factor $e^{-5 / 4 A}$ in front of every field strength in comparison with the IIA case. A discussion of the closure with the conformal group is postponed to the second section. We now give the explicit form of the field strengths. They are given for the scalar by:

$$
\tilde{F}_{a}=e^{-5 / 4 A} D_{a} A
$$

for the 1-form:

$$
\tilde{F}_{a_{1} a_{2}}=2 e^{-5 / 4 A} e^{(3 / 4) A} \tilde{D}_{\left[a_{1}\right.} A_{\left.a_{2}\right]}=2 e^{-5 / 4 A} e^{(3 / 4) A}\left(D_{\left[a_{1}\right.} A_{\left.a_{2}\right]}+\frac{1}{2} m A_{a_{1} a_{2}}\right),
$$

for the 2-form:

$$
\tilde{F}_{a_{1} a_{2} a_{3}}=3 e^{-5 / 4 A} e^{-(1 / 2) A} D_{\left[a_{1}\right.} A_{\left.a_{2} a_{3}\right]}
$$

for the 3-form:

$$
\tilde{F}_{a_{1} \cdots a_{4}}=4 e^{-5 / 4 A} e^{(1 / 4) A}\left(D_{\left[a_{1}\right.} A_{\left.a_{2} \cdots a_{4}\right]}+6 A_{\left[a_{1}\right.} D_{a_{2}} A_{\left.a_{3} a_{4}\right]}+\frac{3}{2} m A_{\left[a_{1} a_{2}\right.} A_{\left.a_{3} a_{4}\right]}\right)
$$

for the 5-form:

$$
\tilde{F}_{a_{1} \cdots a_{6}}=6 e^{-5 / 4 A} e^{-(1 / 4) A}\left(D_{\left[a_{1}\right.} A_{\left.a_{2} \cdots a_{6}\right]}+20\left(D_{\left[a_{1}\right.} A_{a_{2} \cdots a_{4}}+\frac{1}{2} m A_{\left[a_{1} a_{2}\right.} A_{a_{3} a_{4}}\right) A_{\left.a_{5} a_{6}\right]}\right)
$$

for the 6-form:

$$
\begin{aligned}
& \tilde{F}_{a_{1} \cdots a_{7}}=7 e^{-5 / 4 A} e^{(1 / 2) A}\left(D_{\left[a_{1}\right.} A_{\left.a_{2} \cdots a_{7}\right]}+\frac{1}{7} m A_{a_{1} a_{2} \cdots a_{7}}-20 A_{\left[a_{1} a_{2} a_{3}\right.} D_{a_{4}} A_{\left.a_{5} a_{6} a_{7}\right]}+\right. \\
+ & \left.12 A_{\left[a_{1}\right.}\left(D_{\left[a_{2}\right.} A_{\left.a_{3} \cdots a_{7}\right]}+20 A_{a_{2} a_{3}}\left(D_{\left[a_{4}\right.} A_{\left.a_{5} a_{6} a_{7}\right]}+\frac{1}{2} m A_{a_{4} a_{5}} A_{\left.a_{6} a_{7}\right]}\right)\right)\right)
\end{aligned}
$$

for the 7-form:

$$
\begin{gathered}
\tilde{F}_{a_{1} \cdots a_{8}}=8 e^{-5 / 4 A} e^{-(3 / 4) A}\left(D_{\left[a_{1}\right.} A_{\left.a_{2} \cdots a_{8}\right]}-42 A_{\left[a_{1} a_{2}\right.}\left(D_{a_{3}} A_{\left.a_{4} \cdots a_{8}\right]}+10 D_{a_{3}} A_{a_{4} a_{5} a_{6}} A_{\left.a_{7} a_{8}\right]}+\right.\right. \\
\left.\left.+\frac{5}{2} m A_{a_{3} a_{4}} A_{a_{5} a_{6}} A_{\left.a_{7} a_{8}\right]}\right)\right)
\end{gathered}
$$


for the 8-form:

$$
\begin{gathered}
\tilde{F}_{a_{1} \cdots a_{9}}=9 e^{-5 / 4 A}\left(D_{\left[a_{1}\right.} A_{\left.a_{2} \cdots a_{9}\right]}-24 A_{\left[a_{1}\right.} D_{a_{2}} A_{\left.a_{3} \cdots a_{9}\right]}+56 A_{\left[a_{1} a_{2}\right.} D_{a_{3}} A_{\left.a_{4} \cdots a_{9}\right]}-\right. \\
-56 A_{\left[a_{1} a_{2} a_{3}\right.} D_{a_{4}} A_{\left.a_{5} \cdots a_{9}\right]}+1008 A_{\left[a_{1}\right.} A_{a_{2} a_{3}} D_{a_{4}} A_{\left.a_{5} \cdots a_{9}\right]}+8 m A_{\left[a_{1} a_{2}\right.} A_{\left.a_{3} \cdots a_{9}\right]}+ \\
+\frac{7 !}{2} m A_{\left[a_{1}\right.} A_{a_{2} a_{3}} A_{a_{4} a_{5}} A_{a_{6} a_{7}} A_{\left.a_{8} a_{9}\right]}+\frac{8 !}{4} A_{\left[a_{1}\right.} A_{a_{2} a_{3}} A_{a_{4} a_{5}} D_{a_{6}} A_{\left.a_{7} a_{8} a_{9}\right]}- \\
\left.-1120 A_{\left[a_{1} a_{2}\right.} A_{a_{3} a_{4} a_{5}} \tilde{D}_{a_{6}} A_{\left.a_{7} a_{8} a_{9}\right]}-\frac{5}{36} m A_{a_{1} a_{2} \cdots a_{9}}\right)
\end{gathered}
$$

and finally for the 9-form:

$$
\begin{gathered}
\tilde{F}_{a_{1} \cdots a_{10}}=10 e^{-5 / 4 A} e^{-(5 / 4) A}\left(D_{\left[a_{1}\right.} A_{\left.a_{2} \cdots a_{10}\right]}-144 A_{\left[a_{1} a_{2}\right.} D_{a_{3}} A_{\left.a_{4} \cdots a_{10}\right]}+\right. \\
+3024 A_{\left[a_{1} a_{2}\right.} A_{a_{3} a_{4}} D_{a_{5}} A_{\left.a_{6} \cdots a_{10}\right]}+\frac{9 !}{18} A_{\left[a_{1} a_{2}\right.} A_{a_{3} a_{4}} A_{a_{5} a_{6}} D_{a_{7}} A_{\left.a_{8} a_{9} a_{10}\right]}+ \\
\left.+3024 m A_{\left[a_{1} a_{2}\right.} A_{a_{3} a_{4}} A_{a_{5} a_{6}} A_{a_{7} a_{8}} A_{\left.a_{9} a_{10}\right]}\right)
\end{gathered}
$$

where $D_{a}$ is the covariant derivative

$$
D_{a} A_{a_{1} \ldots a_{p}}=e_{a}{ }^{\mu}\left(\partial_{\mu} A_{a_{1} \ldots a_{p}}+\left(e^{-1} \partial_{\mu} e\right)_{a_{1}}{ }^{c} A_{c a_{2} \ldots a_{p}}+\ldots\right)
$$

and $\ldots$ indicates the terms where $\left(e^{-1} \partial_{\mu} e\right)$ acts on the other indices of the gauge field. Also, we have written the exponential $e^{-5 / 4 A}$ separately in front of every field strengths to indicate that it is common to all of them.

Using the Cartan forms which transform only under the local Lorentz group in a manner that their indices suggest we must write down a set of invariant equations. If we ask that they be first order in derivatives they can only be given by

$$
\tilde{F}^{a_{1} \cdots a_{p}}=\frac{1}{(10-p) !} \epsilon^{a_{1} \cdots a_{10}} \tilde{F}_{a_{p+1} \cdots a_{10}}, \quad p=1,2,3,4 .
$$

Here we see that the common exponential factor $e^{-5 / 4 A}$ indeed peels off each equation. The nine form gauge field does not possess a dual field, however, its ten form field strength can be taken to be a constant $m$ times the epsilon symbol

$$
m=e^{-5 / 4 A} \frac{1}{10 !} \epsilon^{a_{1} \cdots a_{10}} \tilde{F}_{a_{1} \cdots a_{10}} .
$$

All the above equations of motion and the Einstein equation are equivalent to those one can derive from the Lagrangian formulation given at the beginning of this chapter. We can see that the simple field strengths of equations (1.5),(1.6) indeed match with those given in our group approach of equations (1.25)-(1.27) and one can indeed verify that the above 
equations of motion for the gauge sector (1.34) and (1.35) are the same as those one can derive from the Lagrangian (1.4) once we take $m$ to be a dynamical field.

To recover the massless case, we simply switch off the commutators with the momentum generator by setting $m=0$. However, because $c_{-1}=m b_{0}$ we also have $c_{-1}=0$. Then using the Jacobi relation

$$
c_{-1}=-c_{9}
$$

we deduce $c_{9}=0$, and then as

$$
c_{2,7} c_{9}=c_{2,7}\left(c_{2}+c_{7}\right)
$$

we also need $c_{2,7}=0$. Thereby the nine form is made redundant $\left(\tilde{F}_{(10)}=d A_{(9)}\right)$ and we are indeed left with the massless case.

\section{Closure with the Conformal Group}

The massive IIA supergravity theory is the non-linear realisation of the group that is the closure of the $G_{m I I A}$ group given above with the ten-dimensional conformal group. The closure of these two groups is an infinite dimensional group, but rather than working with this group we can perform a simultaneous realisation of the $G_{m I I A}$ and the conformal group. What this actually means is that we construct the equations of motion only from combinations of the Cartan forms of the $G_{m I I A}$ group, given above, that can be rewritten in terms of the Cartan or other covariant forms of the conformal group. In doing this one gains invariance under both conformal group and $G_{m I I A}$, and so necessarily we find invariance under the group which is the closure of $G_{m I I A}$ and the conformal group. This is discussed at length in reference [15], but here we briefly discuss the novel features that arise in this procedure when applied to the massive IIA theory.

The two groups only have one Goldstone boson in common namely the trace of $h_{a}{ }^{b}$ which is related to the conformal field $\sigma$. In fact we have to identify these two fields via $e_{\mu}{ }^{a} \equiv\left(e^{h}\right)_{\mu}{ }^{a}=\left(e^{\bar{h}+\delta \sigma}\right)_{\mu}{ }^{a}$ (as in [15]). All the other fields that occur as Goldstone bosons in the $G_{m I I A}$ algebra are viewed as matter fields from the conformal group viewpoint. The conformal covariant derivative of a field $B$ transforming under a representation $\Sigma$ of the Lorentz group is:

$$
\Delta_{\mu} B=e^{-\sigma}\left(\partial_{\mu}+\partial^{\nu} \sigma \Sigma_{\mu \nu}\right) B
$$

In contrast, the $G_{m I I A}$ covariant derivative of a matter field is given by

$$
\tilde{D}_{a} B=\left(e^{-1}\right)_{a}^{\mu}\left(\partial_{\mu}+\frac{1}{2} \omega_{\mu b}{ }^{c} \Sigma^{b}{ }_{c}\right) B
$$

multiplied by a suitable exponential of $A$. As the latter is a Lorentz scalar it plays no part for the discussion of the closure with the conformal group given in this section. Solving 
(2.1) for $\partial_{\mu} B$ and plugging the result into (2.2), we get

$$
\tilde{D}_{a} B=\left(e^{-1}\right)_{a}{ }^{\mu}\left(e^{\sigma} \Delta_{\mu} B-\partial^{\nu} \sigma \Sigma_{\mu \nu} B+\frac{1}{2} \omega_{\mu b}{ }^{c} \Sigma^{b}{ }_{c} B\right) .
$$

If we demand that the whole $\sigma$ dependence be through the conformal covariant derivative, then we learn from this equation that $\omega_{\mu b} c$ must be solved for by a $G_{m I I A}$ invariant condition on the Cartan forms in such a way as to cancel the derivatives of $\sigma$ on the right hand side. This is carried out below and we find that the usual expression of the spin connection in terms of the vielbein (stated below in (2.8)) has precisely the right form to do the job.

We now illustrate the procedure of taking the closure of the conformal and the $G_{m I I A}$ group by considering the vector field $A_{a}$ instead of a general matter field $B$. The other gauge fields are treated in a very similar way. The conformal covariant derivative of a vector (2.1) is given by (see also [15])

$$
\Delta_{\mu} A_{a}=e^{-\sigma}\left(\partial_{\mu} A_{a}+\eta_{\mu a} \partial^{c} \sigma A_{c}-\partial_{a} \sigma \delta_{\mu}^{c} A_{c}\right)
$$

Using this equation we may rewrite the $G_{m I I A}$ covariant derivative of $A_{a}$ given in equation (1.25), respecting (1.33) as

$$
\begin{gathered}
\tilde{D}_{a_{1}} A_{a_{2}}= \\
\bar{e}_{a_{1}}^{\mu}{ }^{\mu}\left(\Delta_{\mu} A_{a_{2}}-e^{-\sigma}\left(\eta_{\mu a_{2}} \partial^{c} \sigma A_{c}-\partial_{a_{2}} \sigma \delta_{\mu}^{c} A_{c}-\partial_{\mu} \sigma A_{a_{2}}-\left(\bar{e}^{-1} \partial_{\mu} \bar{e}\right)_{a_{2}}{ }^{c} A_{c}-\frac{1}{2} m A_{\mu a_{2}}\right)\right),
\end{gathered}
$$

where the vielbein with the overbar stands for the traceless part $e_{\mu}{ }^{a}=e^{\bar{h}_{\mu}{ }^{a}+\delta_{\mu}^{a} \sigma}=\left(\bar{e} \cdot e^{\sigma}\right)_{\mu}{ }^{a}$. We realise that only if we take the combination $\tilde{D}_{[a} A_{b]}$ does the $\sigma$ dependence only appear through the conformal covariant derivative alone, as then the $3 \sigma$-dependent terms in the second bracket vanish since they are symmetric in $\mu$ and $a_{2}$. If we want to use expressions covariant under both the conformal group and $G_{m I I A}$, then we have to demand that all $\sigma$ dependence be implicitly through the conformal derivative alone. As such we conclude that only the totally antisymmetrised object $2 e^{3 / 4 A} \hat{e}_{[a \mid}^{\mu} \tilde{D}_{\mu} A_{\mid b]} \equiv \tilde{F}_{a b}$ is covariant under both groups.

We know that the closure of the $G_{m I I A}$ group and the conformal group generates gauge transformations and general coordinate transformations and so the above object should be covariant under these transformations. We observe that

$$
\tilde{D}_{[a} A_{b]}=2\left(\partial_{[a} A_{b]}+\left(e^{-1} \partial_{[a} e\right)_{b]}^{c} A_{c}+m b_{2} A_{a b}\right)=2 e_{a}^{\mu} e_{b}^{\nu}\left(\partial_{[\mu} A_{\nu]}+m b_{2} A_{\mu \nu}\right),
$$

making it clear that it is covariant under gauge and general coordinate transformations. The $G_{m I I A}$ covariant derivatives of IIA supergravity only differ from those of massive IIA supergravity by terms containing $m$, and the nine form potential. However, as these new terms do not contain derivatives the closure with the conformal group is not spoilt by the presence of these terms. 
We now turn to the gravity sector of the theory. Clearly, the constraint

$$
\Omega_{a,[b c]}-\Omega_{b,(a c)}+\Omega_{c,(a b)}=0 .
$$

is $G_{m I I A}$ covariant, but one can show in much the same way as for the IIA and the eleven dimensional supergravity cases [15] that it is also conformally covariant. Examining the definition of $\Omega_{a, b c}$ in equation (1.21), we see that it involves an undifferentiated factor of $\hat{e}_{a}{ }^{\mu}=e^{-5 / 4 A} e_{a}{ }^{\mu}$. The factor of $e^{-5 / 4 A}$ can then be removed and we find that it is exactly the same constraint as in the other cases (eleven dimensional supergravity, IIA and IIB supergravity) and therefore results in the usual expression for the spin connection in terms of the vielbein, namely

$\omega_{\mu b c}=\frac{1}{2}\left(e_{b}{ }^{\rho} \partial_{\mu} e_{\rho c}-e_{c}{ }^{\rho} \partial_{\mu} e_{\rho b}\right)-\frac{1}{2}\left(e_{b}{ }^{\rho} \partial_{\rho} e_{\mu c}-e_{c}{ }^{\rho} \partial_{\rho} e_{\mu b}\right)-\frac{1}{2}\left(e_{b}{ }^{\lambda} e_{c}{ }^{\rho} \partial_{\lambda} e_{\rho a}-e_{c}{ }^{\lambda} e_{b}{ }^{\rho} \partial_{\lambda} e_{\rho a}\right) e_{\mu}{ }^{a}$.

We conclude that the physical vielbein of general relativity is just $e_{\mu}{ }^{a}$.

The upshot of this discussion is that the simultaneously covariant objects that trans-

form covariantly under the group $G_{m I I A}$ and the conformal group are the $\tilde{F}_{a_{1} \cdots a_{p}}$ for $p=1, \ldots, 10$ (except 5) defined in (1.24)-(1.32) and the Riemann tensor

$$
R_{\mu \nu b}^{c} \equiv \partial_{\mu} \omega_{\nu b}^{c}+\omega_{\mu b}^{d} \omega_{\nu d}^{c}-(\mu \leftrightarrow \nu)
$$

The invariant field equations for all the fields except that of gravity are given in equations (1.34) and (1.35), while that for gravity must be of the form

$$
\begin{gathered}
R_{\mu \nu b}{ }^{c} e_{c}{ }^{\nu} e_{a}^{\mu}=\frac{1}{16} m^{2} e^{5 / 2 A} \eta_{a b}+\frac{1}{2} \partial_{a} A \partial_{b} A+e^{-A}\left(F_{a}^{(3) c d} F_{b c d}^{(3)}-\frac{1}{12} \eta_{a b} F^{(3) c d f} F_{c d f}^{(3)}\right) \\
+2 m e^{3 / 2 A}\left(F_{a}^{(2) c} F_{b c}^{(2)}-\frac{1}{16} \eta_{a b} F^{(2) c d} F_{c d}^{(2)}\right)+\frac{1}{3} e^{1 / 2 A}\left(F_{a}^{(4) c d f} F_{b c d f}^{(4)}-\frac{3}{32} \eta_{a b} F^{(4) c d f g} F_{c d f g}^{(4)}\right) .
\end{gathered}
$$

This equation does not look $G_{m I I A}$ covariant as we seem to have used the unhatted vielbeins only. However, the same factor of $e^{-5 / 2 A}$ turns up in every single term and can therefore again be dropped. We note that, one cannot know the factor in front of each term on the right-hand side. These factors can only be determined if we additionally use information from supersymmetry or the Kac-Moody algebra. We have just put in the correct values for those constants.

\section{Discussion}

We have shown that like all the other maximal supergravity theories the entire bosonic sector of massive IIA supergravity can also be described as a non-linear realisation. Apart from introducing dual fields for all the gauge fields of the original formulation [7] of the 
massive IIA theory we also have included, following [19], a nine form gauge which is associated with the introduction of the cosmological constant. The correct theory requires that the momentum generator has non-trivial commutation relations with the generators associated with the gauge fields as given in equation (1.10). This is natural as the nine form is associated with the cosmological constant and so with gravity.

This is in contrast to the work of reference [14] which takes a different approach and does not include gravity, but does introduce a dual form for the nine form gauge field which was called a "minus one form". The properties of this minus one form are not very explicitly spelt out. In effect we find in this paper that the momentum generator plays the role of the generator associated with the "minus one form" of reference [14].

It would be interesting to examine if the non-linear realisation could be extended, in ways explained in reference [17], to be invariant under a Kac-Moody, or Borcherds algebra, and to conjecture what this algebra is. In the previous non-linear realisation of the maximal supergravities the momentum generator has not played a central part in the Kac-Moody algebra that has been identified. However, the non-trivial relations of equations (1.10) imply that this generator must occur in a non-trivial way in the corresponding algebra. Progress in this direction may also shed light on the place that the massive IIA theory has in $\mathrm{M}$ theory.

\section{Acknowledgements:}

IS would like to thank André Miemiec, who has given support when calculating the equations of motion for various SUGRAs. IS is also supported by DAAD (D/00/09914).

\section{References}

[1] W. Nahm, "Supersymmetries and their representations", Nucl. Phys. B135 (1978), p.149

[2] E. Cremmer, B. Julia, and J. Scherk, "Supergravity theory in 11 dimensions", Phys. Lett. B76 (1978) 409-412.

[3] I. C. G. Campbell and P. C. West, " $N=2 d=10$ nonchiral supergravity and its spontaneous compactification", Nucl. Phys. B243 (1984) 112.; M. Huq and M. Namazie, "Kaluza-Klein supergravity in ten dimensions", Class. Q. Grav. 2 (1985).; F. Giani and M. Pernici, " $N=2$ supergravity in ten dimensions", Phys. Rev. D30 (1984) 325.

[4] J. H. Schwarz and P. C. West, "Symmetries and transformations of chiral N=2,D = 10 supergravity," Phys. Lett. B126 (1983) 301.

[5] J. H. Schwarz, "Covariant field equations of chiral N=2 D = 10 supergravity," Nucl. Phys. B226 (1983) 269.

[6] P. S. Howe and P. C. West, "The complete $\mathrm{N}=2, \mathrm{~d}=10$ supergravity," Nucl. Phys. B238 (1984) 181. 
[7] L. J. Romans, "Massive N=2a Supergravity in Ten Dimensions," Phys. Lett B169 (1986) 374.

[8] S. Ferrara, J. Scherk and B. Zumino, "Algebraic Properties of Extended Supersymmetry", Nucl. Phys. B121 (1977) 393; E. Cremmer, J. Scherk and S. Ferrara, "SU(4) Invariant Supergravity Theory", Phys. Lett. B74 (1978) 61.

[9] E. Cremmer and B. Julia, "The $N=8$ supergravity theory. I. The Lagrangian", Phys. Lett. B80 (1978) 48

[10] B. Julia, "Group Disintegrations", in Superspace 8 Supergravity, p. 331, eds. S.W. Hawking and M. Roček, Cambridge University Press (1981).

[11] H. Nicolai, Phys. Lett. B187 (1987) 316.

[12] C. M. Hull, P. K. Townsend, "Unity of Superstring Dualities," Nucl. Phys. B438 (1995), 109, hep-th/9410167.

[13] E. Cremmer, B. Julia, H. Lü, and C. N. Pope, "Dualisation of dualities. I $\&$ II: Twisted self-duality of doubled fields and superdualities, " Nucl. Phys. B535 (1998) 242 , hep-th/9806106

[14] I. V. Lavrinenko, H. Lü, C. N. Pope, K. Stelle, "Superdualities, Brane Tensions and Massive IIA/IIB Duality," Nucl.Phys. B555 (1999) 201, hep-th/9903057

[15] P. C. West, "Hidden superconformal symmetry in M theory", JHEP 08 (2000) 007, hep-th/0005270

[16] I. Schnakenburg, P. West, "Kac-Moody Symmetries of IIB Supergravity,", Phys. Lett. B517 (2001) 421, hep-th/0107181

[17] P. West, "E(11) and M theory, Classical and Quantum Gravity, 18 (2001) 4443, hepth/0104081

[18] E. Witten, String theory dynamics in various dimensions, Nucl. Phys. B443 (1995) 85 , hep-th 9503124

[19] E. Bergshoeff, M. de Roo, M. B. Green, G. Papadopoulos, P. K. Townsend "Duality of Type II 7-branes and 8-branes," Nucl. Phys. B470 (1996) 113, 9601150 